\title{
Preoperative Incentive Spirometry for Preventing Postoperative Pulmonary Complications in Patients Undergoing Coronary Artery Bypass Graft Surgery: A Prospective, Randomized Controlled Trial
}

\section{Essa M Sweity ( $\sim$ e.sweity@najah.edu )}

An-najah National University Faculty of Medicine and Health Sciences https://orcid.org/0000-00032569-7708

Aidah A Alkaissi

An-najah National University Faculty of Medicine and Health Sciences

Wafiq Othman

An-Najah National University hospital

Ahmad Salahat

An-Najah National University

\section{Research article}

Keywords: incentive spirometry, postoperative pulmonary complication, atelectasis, oxygenation, ventilation time, coronary artery bypass grafting, CABG, length of stay.

Posted Date: May 11th, 2021

DOI: https://doi.org/10.21203/rs.3.rs-483729/v1

License: (c) (i) This work is licensed under a Creative Commons Attribution 4.0 International License.

Read Full License 


\section{Abstract}

Background: Postoperative pulmonary complications (PPCs) often occur after cardiac operations, and are a leading cause of morbidity, inhibit oxygenation, and increase hospital length of stay, and mortality. Although clinical evidence for PPCs prevention is often unclear and crucial, measures take place to reduce PPCs. One device usually used for this reason is the incentive spirometry (IS). The Aim of the study is to evaluate the effect of preoperative incentive spirometry to prevent postoperative pulmonary complications, improve postoperative oxygenation, and decrease hospital stay following coronary artery bypass graft (CABG) surgery patients.

Methods: This was a clinical randomized prospective study. A total of 80 patients were selected as candidates for CABG at An-Najah National University Hospital, Nablus-Palestine. Patients had been randomly assigned into two groups: incentive spirometry group (IS), SI performed before surgery (study group) and control group, preoperative spirometry was not performed. The 40 patients in each group received the same protocol of anesthesia and ventilation in the operating room.

Result: The study findings showed that there was a significant difference between the IS group and control group in the incidence of postoperative atelectasis, there were 8 patients (20.0\%) in IS group and 17 patients $(42.5 \%)$ in the control group $(p=0.03)$. Mechanical ventilation duration was significantly less in group IS group, the median was four hours versus six hours in the control group $(p<0.001)$. Hospital length of stay was significantly less in group IS group, the median was six days versus seven days in the control group ( $p<0.001)$. Median of the amount of arterial blood oxygen and oxygen saturation was significantly effective improvement in IS group with $(p<0.005)$.

Conclusion: Preoperative incentive spirometry for 2 days along with exercises of deep breathing, encouraged coughing and early ambulation following CABG are in connection with prevention and decrease incidence of atelectasis, hospital stay, mechanical ventilation duration and improved postoperative oxygenation with better pain control. A difference that can be considered both significant and clinically relevant.

\section{Trial registration}

Thai Clinical Trials Registry: TCTR20201020005. Registered 17 October 2020 - Retrospectively registered.

\section{Introduction}

Coronary artery disease (CAD) is the leading cause of death and disability worldwide[1]. Coronary artery bypass grafting (CABG) is indicated for patients with angina and suitable coronary anatomy, especially those with stenosis of the left main coronary artery or patients with multi-vessel disease[2].

Postoperative pulmonary complications (PPCs) are a frequent incident following cardiac, thoracic and abdominal surgeries[3]. PPCs are very common following CABG surgery and the incidence is between 
$30 \%$ and $60 \%$ [4]. PPC complications contribute significantly to morbidity, mortality and hospitalization costs[5]. These complications include atelectasis, pulmonary infections such as pneumonia and bronchitis, pleural effusion, pulmonary edema, and respiratory insufficiency[6].

Atelectasis is a highly prevalent complication following coronary artery bypass graft (CABG) surgery[7]. There is no clear cause yet for atelectasis, but several factors may contribute, such as impairment in the function of the diaphragm, general anaesthesia, 'abdominal distension, chest wall shift, pain and pleural effusions[8].

The pain and postoperative fear associated with changes in lung mechanics resulting from the surgery affect the performance of periodic deep inspiration and effective cough, allowing the accumulation of secretion, alveolar collapse and changes in gas exchange[9].

Shaban et al.[10] reported that pre-operative breathing exercise decreases the incidence of atelectasis, improves ventilation status and decreases hospital stay. Also Oshvandi et al.[11]. verified that atelectasis postoperatively can be decreased by obtaining several deep breath plus 3 seconds holding the breath concurrent with IS, while Freitas et al. revealed that there is no benefit of IS in decreasing PPCs in patients following CABG surgery[12] .

Although clinical evidence regarding PPC prevention is often unclear, crucial measures take place to reduce PPCs. These include carefully individualized strategies for preventing atelectasis and aspiration of oral secretions, increasing the patient's ability to mobilize, expectorate secretions and restore functional residual capacity[13]. There are several measures applied for preventing PPCs, such as deep-breathing exercises, IS, early ambulation and positive airway pressure[14, 15].

Incentive spirometry (IS) is one tool frequently used for this purpose[3]. The IS is a handheld device used postoperative to reach effective inspiration. Patients practicing IS provide visual and positive feedback after inhaling at a determined flow or volume rate and holding the inflation for at least 3 seconds[16]. IS intended to mimic normal sighing or yawning by supporting the patient to take long and slow deep breaths. This reduces pleural tension, supporting enhanced lung expansibility and improving ventilation perfusion. While practices of IS is being on an ordered basis, atelectasis may be avoided or reduced[17].

IS was found to decrease the incidence of PPCs and length of stay after upper abdominal surgery [18]. By contrast, many study publications have questioned its effectiveness[19, 20].

Monitoring, instruction and teaching the patient how to use the IS are the responsibility of nursing and respiratory therapy staff. Respiratory therapy that involves periods of IS each day in addition to deepbreathing applications, guided coughing, early mobilization and pain control can reduce the incidence of PPCs[21] Incentive spirometry may prevent PPCs in patients following CABG surgery[8].

Applications of deep breathing are shown to reduce the occurrence and severity of PPCs, such as pneumonia and atelectasis. Through application instruction, the nurse clarifies and displays how to take a deep and gradual breath, also how to exhale gradually, three to five times every 1 to 2 hours. Patients 
who carried out deep-breathing exercises had improved pulmonary function in contrast with nonpracticing groups[22].

Afrasiabi et al. conducted a study about the influence of IS on the oxygenation status of arterial blood gases following a CABG operation. Throughout $6 \mathrm{~h}$ after extubation, patient was handled, the IS and preoperative, $1 \mathrm{~h}$, and $7 \mathrm{~h}$ after extubation arterial blood gases $[\mathrm{R}]$ obtained. The researcher revealed there was no significant benefit in oxygenation status measured by ABG's after using IS[23]. Freitas, et al, Carvalho et al, Eltorai et al, and Overend et al., have declared that, to date, there is no evidence to support the practice of IS to decrease PPCs. although IS is still usually requested to reduce PPCs, despite the narrow evidence to support its advantages and the absence of a harmonized protocol and they recommend that additional research is necessary to clarify this issue[12, 19, 20,24]. Agostini and Singh, differ from this opinion and have stated that this practice can improve pulmonary function[25].

Preoperative education gives health-related information for patients, which prepares them for surgery and helps to decrease the development of PPCs[26]. In numerous studies it is suggested that postoperative incentive spirometry is practiced to decrease PPCs and decrease length of stay (LOS), but the success of postoperative incentive spirometry is dependent not only on the postoperative, but also the preoperative period, which has been shown to improve oxygenation, decrease the incidence of PPCs and to decrease hospital LOS[27-29]. Another study has shown that the rate of pneumonia and atelectasis reduced with breathing exercise and IS in obese patients prior to CABG surgery[30]. by contrast Moradian et al [31]revealed that preoperative breathing exercise with incentive spirometry does not reduce pulmonary complications and hypoxemia. IS training before and after the operation significantly improved lung inspiratory capacity and arterial oxygenation in CABG patients[32].

Since PPCs exhibit elevated rates of hospital costs, morbidity, mortality, and increased length of hospital stay following CABG surgery, it is evident that it is essential to discuss the use of IS preoperatively to reduce PPCs and to decrease post-operative length of stay in the intensive coronary care unit (ICCU) and in the hospital. The aim of the study is to evaluate the effect of preoperative incentive spirometry in preventing postoperative pulmonary complications, improving postoperative oxygenation and decreasing length of stay at hospital in patients following CABG surgery.

\section{Materials And Methods}

\section{Study design}

The study was conducted as prospective, cohort, randomized controlled trial (RCT). This design was adopted due the strength of the hierarchy of scientific evidence, namely, reduced bias and more accurate results.

\section{Study Setting and Population}


The study was conducted at AN Najah National University Hospital. Data was collected from CCU and Intermediate CCU wards. An-Najah National University Hospital has 200 beds, 5 beds for CCU and 16 beds for Intermediate CCU. It is a non-profit hospital, located in the Northern West Bank, Palestine.

Participants are adult patients scheduled for coronary artery bypass surgery, aged 18 or older, and patients who were well motivated and compliant.

\section{Sample and sampling}

To investigate the optimal sample magnitude for the trial that safeguards an adequate effect to identify statistical significance, the effect of the trial was estimated at 80 percent power, with alpha levels at ( $p$ $\leq 0.05$ ). Sample magnitude was computed as 37 patients for each group by using A formula (i.e., Pocock's sample size formula) that can be directly applied for comparison of proportions P1 and P2 in two equally sized groups. To raise the potential of the current trial, we recruited 40 patients in every group as has also been done in early studies.

\section{Inclusion and exclusion criteria}

Inclusion :18 years or older, scheduled to have coronary artery bypass grafting (CABG) and patients who were well motivated and compliant.

Exclusion: Patients who are expected not to be able to conduct or comply with IS, such as patients with cognitive or neurological deficits, patients with coexisting acute or chronic respiratory disorders, patients unable to understand or show the proper use of the incentive spirometer, patients who cannot be instructed or supervised to assure appropriate use of the device, patients in whom cooperation is absent or patients unable to understand or demonstrate proper use of the device, patients who are confused or delirious, patients undergoing any other surgery along with $C A B G$, having prolonged mechanical ventilation (more than 24 hours) or re-intubation, patients undergoing emergency CABG surgery, chronic obstructive pulmonary disease (COPD), asthma, restrictive lung disease, preoperative major chest infection e.g. pulmonary tuberculosis, chest deformities such as pectus carinatum, pectus excavatum, thoracolumbar scoliosis, diaphragmatic hernias diagnosed on history.

\section{Randomization and Blindness}

The participants who met the inclusion criteria were randomized into two groups according to a randomization list formatted by www.randomization.com.

Group 1: Incentive spirometry was utilized by the patient with 10 breaths, 6 times per day for a period of 10 minutes in every session with a breathing technique for 2 days preoperatively. The patients were taught how to use IS by a nurse who would not be involved in the patient's postoperative care. (Experimental group) (IS).

Group 2: No IS preoperatively, only IS postoperatively (Control Group) 
The patients, health care providers included in the patient care are unaware of the treatment group allocation.

\section{Measured Outcomes:}

The primary outcome was defined as respiratory complications that occur within $48-72 \mathrm{~h}$ following surgery, which include: atelectasis, pneumonia, pleural effusion, and Pneumothorax. Which measured by $x$-ray and clinical sign and symptom[33] . Secondary outcomes included hospital length of stay that calculated by subtracting day of admission from day of discharge[34]. Mechanical ventilation duration in hours, oxygenation status by measuring $\mathrm{PaO} 2$ and $\mathrm{SaO} 2$ by ABG's, and control pain by using numerical pain scale.

\section{Measurement and data collection procedure}

The participants who met the inclusion criteria and randomized to the intervention group were given a flow-based incentive spirometer and asked to use spirometry with deep-breathing exercise 2 days preoperatively until surgery. They were asked to hold the spirometer in the upright position, place their lips tightly across the spirometer mouthpiece, and then they were asked to slowly inhale air into the lungs to raise the ball to the target position. After that, the mouthpiece was removed and patients were asked to hold their breath for at least 5 seconds followed by normal expiration. Incentive spirometry was done with 10 breaths, 6 times per day for a period of 10 minutes every session before surgery according to literature and An-Najah National University hospital.

Observations and hemodynamic parameters were measured preoperatively and postoperatively. For both groups, study observations were recorded every 6 hours. Both groups postoperative received the same intervention: the exercises began on the morning after surgery with incentive spirometry, deep-breathing exercise and physiotherapy after Extubation, and early mobilization, in accordance with An-Najah University Hospital protocol. A data sheet containing the following information was filled out for each patient: name, age, height, weight, body mass index, respiratory status, medical history, presence of respiratory complications, duration of $\mathrm{MV}$, and numerical pain scale and hospital length of stay.

The data collection sheet was prepared after going throughout the linked literature and with the supervision of experts in the field. Content validity is defined as "the degree to which objects in an instrument reflect the content universe to which the instrument will be generalized [35]. Content validity was applied while the data sheet was developed to ensure that it included all items that was essential [36, 37]. The assessment method for determining the validity of the data sheet included literature reviews and then follow-ups with evaluation by expert judges or panels (two intensivists, one anaesthesiologist, and three nurses in critical care), all experts' suggestions were taken into account.

Thoracic x-rays were taken during the preoperative period, as well as immediately following surgery in the intensive cardiac care unit (ICCU), on the ward, once the drains had been removed (48 hours after 
surgery), and on discharge from hospital. X-ray examinations were performed at the same frequency for all patients in both groups.

\section{Anaesthesia protocol}

(AN-Najah National University Hospital protocol)

All patients in both groups received the same anaesthesia technique and ventilation in the operation room.

A standard induction for cardiac anaesthesia started with inhalation of sevoflurane $0-8 \%$ in $100 \%$ oxygen and fresh gas flow of $3 \mathrm{~L} / \mathrm{min}$ for 5 min with a facial mask. After that, patients were given IV anaesthesia Propofol $2 \mathrm{mg} / \mathrm{kg}$ IV and tracheal intubation was facilitated with rucoronium 1.5 to $2 \mathrm{mg} / \mathrm{kg}$ with fentanyl $2-20 \mathrm{mcg} / \mathrm{kg} /$ dose initially.

\section{Maintenance of anaesthesia}

Anaesthesia was maintained with sevoflurane $0-3 \%$ in $50 \%$ oxygen and $50 \%$ air. Neuromuscular blockade was maintained with increments of atracurium with this equation: 0.3 (dose) ${ }^{\star} \mathrm{kg} / 4$ (concentration $/ \mathrm{ml}$ ) $=\mathrm{ml} / \mathrm{hr}$, fentanyle was used to provide intraoperative analgesia with this equation: 2 (dose) ${ }^{*} \mathrm{~kg} / 20$ (concentration $/ \mathrm{ml}$ ) $=\mathrm{ml} / \mathrm{hr}$ as $1-2 \mathrm{mcg} / \mathrm{kg} / \mathrm{hr}$ maintenance.

For special cases like decreased ejection fraction and left main coronary disease, etomidate $0.3-0.6$ $\mathrm{mg} / \mathrm{kg}$ was used.

\section{Ethical considerations}

The institutional Review Board (IRB) of An-Najah National University approved the study. Consent forms were obtained from the patients prior to participation and the study was registered in Thai Clinical Trials Registry (no. TCTR20201020005). All patients were given both verbal and written information about the aim and objectives of the study before considering participation in the study. The study followed the World Health Organization Declaration on the Ethical Principles of Helsinki for Medical Research on Humans[38].

\section{Analyses}

The data were analysed with SPSS version 22 for Windows (IBM Corp., Armonk, NY, USA). Data normality was tested using Kolmogorov-Smirnov test. The data were not normally distributed. Thus, nonparametric statistics tests were used. The Scale data are expressed as the median (quartile 1 [Q1]quartile 3 [Q3]). The groups were compared with the Mann-Whitney U Test. Categorical variables (YES/NO questions) were statistically analysed with Chi-square tests have been used. A P value $\leq 0.05$ was considered to indicate a statistically significant difference. 


\section{Results}

One hundred clients were assessed for eligibility, but 20 were excluded, 15 of them not meeting the inclusion criteria, three declined to participate, and two converted to PCl. The patients who did not meet the criteria switched to the hospital routine (using incentive spirometry with deep-breathing exercise postoperatively only). The remaining 80 clients were enrolled in the study and randomly allocated into two groups as shown in Consort diagram (Figure 1).

\section{Socio-demographic and medical history characteristics of the study participants}

Demographic data were comparable between the two groups (Table 1). All patients in the two groups were comparable in terms of age, gender, comorbidity, smoking, and BMI. Hemodynamic parameters and other observations were recorded before operation, postoperatively, and 3 days postoperatively and there are no significant differences between the IS Group and the Control Group in all general characteristics of patients exhibited in the table at the 0.05 level ( $p$-values>0.05).

Table 1: Demographic data and history. 


\begin{tabular}{|c|c|c|c|c|}
\hline Variable & Total $($ Mean \pm SD $)$ & $\begin{array}{l}\text { IS Group } \\
\text { (Mean } \pm \text { SD) }\end{array}$ & $\begin{array}{l}\text { Control Group } \\
\text { (Mean } \pm \text { SD) }\end{array}$ & $P$ value \\
\hline Age & $54.3 \pm 4.5$ & $54.4 \pm 3.8$ & $54.3 \pm 5.1$ & 0.961 \\
\hline Variable & Yes/ No & IS Group n (\%) & Control Group n (\%) & $P$ value \\
\hline \multirow[t]{2}{*}{ Gender } & Male & $22(55.0 \%)$ & $21(52.5 \%)$ & \multirow[t]{2}{*}{0.823} \\
\hline & Female & $18(45.0 \%)$ & $19(47.5 \%)$ & \\
\hline \multirow[t]{2}{*}{ DM } & Yes & $17(42.5 \%)$ & $20(50.0 \%)$ & \multirow[t]{2}{*}{0.501} \\
\hline & No & $23(57.5 \%)$ & $20(50 \%)$ & \\
\hline \multirow[t]{2}{*}{ HTN } & YES & $15(37.5 \%)$ & 15 (37.5\%) & \multirow[t]{2}{*}{$>0.999$} \\
\hline & NO & $25(62.5 \%)$ & 25 (62.5\%) & \\
\hline \multirow[t]{2}{*}{ IHD } & YES & $18(45.0 \%)$ & $15(37.5 \%)$ & \multirow[t]{2}{*}{0.496} \\
\hline & NO & $22(55.0 \%)$ & $25(62.5 \%)$ & \\
\hline \multirow[t]{2}{*}{$\mathrm{PCl}$} & YES & $5(12.5 \%)$ & $8(20.0 \%)$ & \multirow[t]{2}{*}{0.363} \\
\hline & NO & $35(87.5 \%)$ & $32(80.0 \%)$ & \\
\hline \multirow[t]{2}{*}{ SMOKING } & YES & $14(35.0 \%)$ & $16(40.0 \%)$ & \multirow[t]{2}{*}{0.644} \\
\hline & NO & $26(65.0 \%)$ & $24(60.0 \%)$ & \\
\hline BMI & & $26.5 \pm 2.6$ & $26.4 \pm 2.1$ & 0.967 \\
\hline BMI & Normal weight & $12(30.0 \%)$ & $9(22.5 \%)$ & \multirow{3}{*}{0.727} \\
\hline \multirow[t]{2}{*}{ CATEGORY } & Overweight & $24(60.0 \%)$ & $28(70.0 \%)$ & \\
\hline & Obesity & $4(10.0 \%)$ & $3(7.5 \%)$ & \\
\hline
\end{tabular}

\section{Respiratory complication}

Figure 2 shows the respiratory complications of atelectasis among the IS Group and the Control Group. On operation day, there is no atelectasis diagnosed in either group. On the first day, of the 22 patients with atelectasis, 7 were in the IS Group and 15 in the Control Group, showing statistically significant differences $(P$ value $=0.045)$. On the next day, the number of patients in the Control Group with atelectasis was 17 , and in the IS group it was 8 , with statistically significant differences ( $P$ value $=0.030$ ). Furthermore, the third day shows 4 clients with atelectasis in the Control Group and zero patients in the IS group, with statistically significant differences $(P$ value $=0.040)$. On the other hand, there were no statistically significant differences between the groups in other respiratory complications.

\section{Length of stay:}


Figure 3 show that there are significant differences between the IS Group and the Control Group in length of stay in the intensive cardiac care unit (ICCU), intermediate cardiac care unit(IMCCU) and hospital until discharge with ( $P$ value $=<0.001$ ). The IS Group average was 3 days in ICCU, two and a half days in IMCCU. Whereas, the Control Group average was 4 days in ICCU and 3 days in IMCCU.

\section{Duration of mechanical ventilation}

Figure 4 shows that there are significant differences between the IS Group and the Control Group in the duration of time spent in mechanical ventilation in the intensive cardiac care unit (ICCU). Patients in the IS Group spent 4 hours, while patients in the Control Group spent 6 hours with (P-value $=<0.001)$. The median hours spent was 5 hours.

\section{Oxygenation}

\section{The partial pressure of oxygen (Pao2)}

Figure 5 below show that there were significant differences between the IS Group and the Control Group in the partial pressure of oxygen (Pao2), with obvious improvement in Pao2 in the IS Group, as shown in the $P$ Value from $6 \mathrm{hr}-90 \mathrm{hr}$. On the other hand, there were no significant differences between the groups pre-and postoperatively with (P-value $=0.900$ and 0.149 ), respectively.

\section{Oxygen saturation of arterial blood (SaO2\%)}

Figure 6 show that there are significant differences between the IS Group and the Control Group in the oxygen saturation of arterial blood (Sao2), with obvious differences, as shown in the P Value in all postoperative measurement times. On the other hand, there were no significant differences between the groups preoperatively with $(p$-value $=0.335)$.

\section{Numerical rating scales (NRS) pain scale}

Figure 7 show that there were significant differences between the IS Group and the Control Group in the numerical rating scale (NRS) pain scale, with obvious less pain in the IS Group than the control when using the same analgesic plan, as shown in the $P$ value below in all measurements at all times except at 12 hours. In the NRS, scores $\leq 5$ corresponded to mild, scores of $6-7$ to moderate, and scores $\geq 8$ corresponded to severe pain.

\section{Discussion}

The results of this study indicate that IS used preoperatively for patients with CABG surgery to reduce postoperative atelectasis, length of hospital stay, and improved postoperative oxygenation.

\section{The effect of preoperative incentive spirometry on Atelectasis}


In the current study, there was a significant decrease in the incidence of atelectasis in the IS Group. This finding is consistent with Diken and Özyalçın[30], who conducted an RCT that involved 108 patients divided into two groups: IS preoperative and routine care for control, with a body mass index over 30 $\mathrm{kg} / \mathrm{m} 2$ and without previous pulmonary disease. In Diken and Özyalçın's study, patients with atelectasis were predominantly higher in the Control Group compared to the IS Group (18 vs. 7 patients, respectively) $(P=0.0036)$. The current findings also agree with Gilani et al.[39], who conducted an RCT that showed the incidence of postoperative atelectasis was $14.10 \%$ in Group I (IS) and $27.10 \%$ in Group II patients (control) $(p=0.04)$. Moreover, the current study results are consistent with Oshvandi et al.[11], who were showed that the occurrence of atelectasis, respiratory status, dyspnea and sweating showed a significant difference between the IS and control groups at all hours after surgery $(P<0 / 001)$. Furthermore, the results are also in agreement with Shaban et al.[10], who showed the incidence rate of atelectasis in the experimental group was $(26.7 \%)$, less than control group $(56.7 \%)$ with $(P=0.01)$. In addition, the study results also agree with Nardi et al.[18], who revealed that better clinical results for respiratory and musculoskeletal function were found in the groups preoperatively treated with physiotherapeutic protocols immediately before as well as after cardiac surgery. Just the same results were confirmed by Yánez-Brage et al[8] in their observational study, which was conducted on 263 patients and revealed that preoperative physiotherapy (involving incentive spirometry, deep-breathing exercises, assisted coughing and early ambulation) after off-pump CABG surgery was related to a lower incidence of atelectasis.

Conversely, the current study is inconsistent with a study conducted by Moradian et al [31]. This study examined 100 participants and found no significant differences between the IS and control groups in terms of atelectasis and hypoxemia ( $p$ value>0.05). Freitas, et al.[12] also revealed no evidence of a difference between groups in the incidence of PPCs with IS and treatment with physical therapy, positivepressure breathing techniques, active cycle of breathing, or preoperative patient education and worse pulmonary function and arterial oxygenation. Eltorai et al.[24] investigated the clinical effectiveness of incentive spirometry and found that there was narrow evidence to support its advantages and an absence of harmonized protocol for its use. In addition, Overend,et al.[20] conducted a systematic review study and concluded that evidence does not support the use of IS for decreasing the incidence of PPCs.

\section{The effect of preoperative incentive spirometry on oxygenation}

The current results showed a significant improvement in Pao2, Sao2, and SPO2 in the IS Group compared to the Control Group. These results are consistent with Fayyaz et al[27] , where preoperative spirometry had improved postoperative oxygenation in the IS group to 97.29 while the control group was 93.27. Yazdemik, et al.[28] also concluded that incentive spirometry caused a remarkable improvement of Pao2, Sao2 and SP02. Another study conducted by Moradyan et al.[29] corresponds to the current study results and revealed that protocols for breathing exercises (deep breathing, incentive spirometry and directed maneuvers) can improve PaO2 and SaO2. While Balandiuk and Kozlov [32] revealed that the use of incentive spirometer preoperatively for cardiac surgery significantly improved arterial oxygenation. 
Freitas et al.[12] presented results that contradict the current results. They found no differences between the study groups in terms of incentive spirometry used and found poorer lung function and status of arterial oxygenation competed with those treated with positive pressure ventilation. Diken and Özyalçın [30] also disagree with our study results after conducting RCT on two groups and showed no change in oxygenation status for both groups. Even Afrasiabi et al [23] reported that incentive spirometry had no significant effect on improvement in postoperative oxygenation. In addition, Yánez-Brage et al [8] also reported that improvement in postoperative oxygenation using incentive spirometry is not permanent, this improvement is reversible after a short period of time. Carvalho et al [19] in a systematic review study, reviewed 30 studies in relation to IS. They reported that there was no strong evidence to support the use of IS after CABG, and there is a need for studies to clarify the effect and justify the use of this technique.

\section{The effect of preoperative incentive spirometry on hospital length of stay}

The current results showed that the incidence of hospital length of stay for the IS Group was 6 days, while in the Control Group it was 7 days. ICU LOS for the IS Group was also reduced compared with the Control Group. This finding is consistent with Nardi et al [18] who revealed that the hospital stay was further reduced for the IS group. In addition, Shaban et al [10] reported the same results when they declared that the hospital length of stay decreased for the IS group compared to the control group. On the other hand, Fayyaz et al [27]presented results that contradict the current results. They revealed that there were no differences between the groups in length of hospital stay.

\section{The effect of preoperative incentive spirometry on mechanical ventilation time}

The current results showed significant differences between the two groups (IS and Control) regarding mechanical ventilation time (duration), which was 4 hours for the IS Group and 6 hours for the Control Group. This finding is consistent with Gilani et al [39] who showed that mechanical ventilation time was significantly less in Group I patients (IS): it was $5.49+2.28$ hours versus $6.74+5.46$ hours in Group II patients (control) (p-value 0.05). This finding also agrees with Balandiuk and Kozlov [32], who reported that a significant decrease in the duration of MV in the IS group was 7.3 hours compared to 10.4 hours ( $P$ $<0.05)$ in the control group. Moradian et al [31] presented results that contradict the current study results. They revealed that there were no differences between the groups in mechanical ventilation time, with 10.5 hours for the IS group and 11.5 hours for the control group. Yazdemik et al [28] also reported the same duration of mechanical ventilation in both groups following coronary artery bypass surgery. However, Afrasiabi et al [23] presented results that contradict the current study results. They found no differences in mechanical ventilation time between the study groups. Furthermore, Yánez-Brage et al [8] in an observational study, showed that no statistical differences were observed during the time of mechanical ventilation between the study groups.

\section{The effect of preoperative incentive spirometry on pain control}

The current results showed significant differences between the incentive spirometry group and the control group in the numerical rating scales (NRS) pain scale with obvious pain in IS group rather than control 
with using the same analgesic plan as shown $P$ value in all measurements at all times except at 12 hours. With consider NRS, scores $\leq 5$ correspond to mild, scores of $6-7$ to moderate, and scores $\geq 8$ to severe pain. This finding is agreed with Renault et al [9] who report that pain and postoperative fear associated with changes in lung mechanics that affect the performance of periodic deep inspiration and effective cough with effective spirometry, allowing the accumulation of secretion, alveolar collapse and changes in gas exchange, early incentive with cough decreases pain and better control.

\section{IS clinical application}

The current study demonstrated the clinical application and IS a protocol that showed important results in reducing atelectasis occurrence in CABG patients. Meanwhile, some of clinical trials question the effectiveness of IS use and why it is still prescribed to patients in different locations, especially after cardiac surgery $[21,24]$. This paper suggests the implication about this protocol in the intensive cardiac care units especially with a critical care nurses.

\section{Recommendations}

This study was performed on patients who received invective spirometry for 2 days preoperative and did not have lung problems. Therefore, it is recommended to conduct a clinical study with the aim of examining incentive spirometry with deep breathing and cough trials in patients who will undergo CABG surgery with lung problems such as chronic obstructive pulmonary disease and asthma.

\section{Conclusions}

Preoperative incentive spirometry along with deep breathing exercises, assisted coughing, and early ambulation after coronary bypass surgery is related to the prevention and lower incidence of atelectasis, hospital length of stay, duration of mechanical ventilation and improved postoperative oxygenation. A difference that can be considered both significant and clinically relevant.

\section{Abbreviations}

IS: Incentive spirometry

POLS: Postoperative hospital length of stay

NNUH: AN Najah National University Hospital

PPCs: Postoperative pulmonary complications

CABG: Coronary artery bypass graft

WHO: World Health Organization

IRB: Institutional review board 
SPSS: Statistical Package for the Social Sciences

CAD: Coronary artery disease

IPPB: Intermittent positive-pressure breathing

PaO2: Partial Pressure of Oxygen

Sao2: Invasive Oxygen saturation

Spo2: Non- Invasive Oxygen saturation

PaCo2: Partial pressure of carbon dioxide

ICU: Intensive Care Unit

ECG: Electrocardiogram

RCT: Randomized control trial

MV: Mechanical Ventilator

ABG's: Arterial Blood Gases

OR: Operation Room

IMCCU: intermediate cardiac care unit

NRS: numerical rating scales

\section{Declarations}

\section{Ethics approval and consent to participate}

The institutional Review Board (IRB) of An-Najah National University approved the study with archived number 14. Consent forms were obtained from the patients prior to participation and the study followed the World Health Organization Declaration on the Ethical Principles of Helsinki for Medical Research on Humans.

\section{Consent for publication}

Not applicable.

\section{Availability of data and materials}

Data used to support the findings of this study is presented in the manuscript or available from the corresponding author (Essa M Sweity, E-mail: E.sweity@najah.edu) upon reasonable request. 


\section{Competing interests}

The author(s) declare that they have no competing interests

\section{Funding Statement}

No funding was available, the study was self-financed by the principle author.

\section{Authors' contributions}

EMS: Literature search, preparation of the manuscript, revising the manuscript, submission, corresponding author. AA: Literature search, preparation of the manuscript, Revising the manuscript, WO: Literature search, preparation of the manuscript. AS: Literature search, preparation of the manuscript, revising the manuscript. All authors read and approved the final manuscript.

\section{Author information}

Affiliations: An-Najah National University, Nablus 44839, Palestine.

Faculty of Medicine and Health Sciences, Nursing and Midwifery Department, An-Najah National University, Nablus 44839, Palestine.

\section{Corresponding Author}

Essa M.Sweity

\section{Acknowledgments}

I would like to give my grateful to Dr. Saed Zyoud for his endless help and support in his impactful research suggestions and statistical analysis consultation for this project.

\section{References}

1. WHO, The top 10 causes of death. Fact sheets, 2018. http://www.who.int/news-room/factsheets/detail/the-top-10-causes-of-death.

2. Hillis, L.D., et al., 2011 ACCF/AHA guideline for coronary artery bypass graft surgery: executive summary: a report of the American College of Cardiology Foundation/American Heart Association Task Force on Practice Guidelines developed in collaboration with the American Association for Thoracic Surgery, Society of Cardiovascular Anesthesiologists, and Society of Thoracic Surgeons. Journal of the American College of Cardiology, 2011. 58(24): p. 2584-2614.

3. Branson, R.D., The Scientific Basis for Postoperative Respiratory Care. Respiratory Care, 2013. 58(11): p. 1974-1984.

4. Mullen-Fortino, M., N. O'Brien, and M. Jones, Critical care of a patient after CABG surgery. 2009. 4(4): p. 46-53. 
5. Miskovic, A. and A.B. Lumb, Postoperative pulmonary complications. British Journal of Anaesthesia, 2017. 118(3): p. 317-334.

6. Hulzebos, E.H., et al., Preoperative intensive inspiratory muscle training to prevent postoperative pulmonary complications in high-risk patients undergoing CABG surgery: a randomized clinical trial. Jama, 2006. 296(15): p. 1851-1857.

7. Ferreira, G.M., et al., Espirometria de incentivo com pressão positiva expiratória é benéfica após revascularização miocardio \%J Arquivos Brasileiros de Cardiologia. 2010. 94: p. 246-251.

8. Yánez-Brage, l., et al., Respiratory physiotherapy and incidence of pulmonary complications in offpump coronary artery bypass graft surgery: an observational follow-up study. BMC Pulmonary Medicine, 2009. 9(1): p. 36.

9. Renault, J.A., et al., Comparação entre exercícios de respiração profunda e espirometria de incentivo no pós-operatório de cirurgia de revascularização do miocárdio. Brazilian Journal of Cardiovascular Surgery, 2009. 24: p. 165-172.

10. Shaban, M., et al., Assessment the effects of respiratory exercise education in acute respiratory complication and the length of patient hospitalization, for undergoing coronary artery by-pass surgery in Kermanshah Emam Ali hospital. Journal of hayat, 2002. 8(2): p. 12-20.

11. Oshvandi, K., et al., Effect of Respiratory Exercises on the Prevalence of Atelectasis in Patients Undergoing Coronary Artery Bypass Surgery. Avicenna Journal of Nursing and Midwifery Care, 2020. 27(6): p. 432-440.

12. Freitas, E., et al., Incentive spirometry for preventing pulmonary complications after coronary artery bypass graft. Cochrane Database of Systematic Reviews, 2012(9).

13. David A Grooms MSHS, R. Postoperative Pulmonary Complications. Clinical Foundations, 2012. 13, 1-11.

14. Wynne, R. and M. Botti, Postoperative Pulmonary Dysfunction in Adults After Cardiac Surgery With Cardiopulmonary Bypass: Clinical Significance and Implications for Practice. American journal of critical care : an official publication, American Association of Critical-Care Nurses, 2004. 13: p. 38493.

15. Zarbock, A., et al., Prophylactic Nasal Continuous Positive Airway Pressure Following Cardiac Surgery Protects From Postoperative Pulmonary Complications: A Prospective, Randomized, Controlled Trial in 500 Patients. Chest, 2009. 135(5): p. 1252-1259.

16. Westwood, K., et al., Incentive spirometry decreases respiratory complications following major abdominal surgery. Vol. 5. 2008. 339-42.

17. Anandhi, D. and P. Divya, Influence of Various Factors on the Incentive Spirometry Values in Patients Undergoing Thoracotomy. Annals of Physiotherapy Clinics, 2018. 1(1): p. 1-5.

18. Nardi, P., et al., The effect of preoperative respiratory physiotherapy and motor exercise in patients undergoing elective cardiac surgery: short-term results. Kardiochirurgia i torakochirurgia polska = Polish journal of cardio-thoracic surgery, 2019. 16(2): p. 81-87. 
19. Carvalho, C.R.F., D.M. Paisani, and A.C. Lunardi, Incentive spirometry in major surgeries: a systematic review. Brazilian Journal of Physical Therapy, 2011. 15: p. 343-350.

20. Overend, T.J., et al., The Effect of Incentive Spirometry on Postoperative Pulmonary Complications: $A$ Systematic Review. CHEST, 2001. 120(3): p. 971-978.

21. Restrepo, et al., Incentive spirometry: 2011. Respir Care, 2011. 56(10): p. 1600-4.

22. Ünver, S., G. Kıvanç, and H.M. Alptekin, Deep breathing exercise education receiving and performing status of patients undergoing abdominal surgery. International journal of health sciences, 2018. 12(4): p. 35-38.

23. Afrasiabi, K. Ansarin, and S. Hassanzadeh Salmasi, Comparison of the Effect of Incentive Spirometry on Pulmonary Volumes and Arterial Blood Gases after Coronary Artery Bypass Surgery. Armaghane danesh, 2006. 11(3): p. 1-9.

24. Eltorai, et al., Perspectives on Incentive Spirometry Utility and Patient Protocols. Respiratory Care, 2018. 63(5): p. 519-531.

25. Agostini, P. and S. Singh, Incentive spirometry following thoracic surgery: what should we be doing? Physiotherapy, 2009. 95(2): p. 76-82.

26. GÜRLEK, Ö. and M. YAVUZ, CERRAHI KLINIKLERDE ÇALIŞAN HEMŞIRELERIN AMELIYAT ÖNCESi HASTA EĞiTiMi UYGULAMA DURUMLARI. Anadolu Hemşirelik ve Sağlık Bilimleri Dergisi, 2013. 16(1): p. 8-15.

27. Fayyaz, et al., PRE-OPERATIVE INCENTIVE SPIROMETRY, EFFECTIVENESS TO IMPROVE POSTOPERATIVE OXYGENATION IN PATIENTS UNDERGOING CABG SURGERY. THE PROFESSIONAL MEDICAL JOURNAL, 2016. 23: p. 1368-1372.

28. Yazdannik, A., et al., The effect of incentive spirometry on arterial blood gases after coronary artery bypass surgery (CABG). Iranian journal of nursing and midwifery research, 2016. 21(1): p. 89.

29. Moradyan, T., et al., The effect of planned breathing exercises on oxygenation in patients after coronary artery bypass surgery. Iranian Journal of Cardiovascular Nursing, 2012. 1(1): p. 8-14.

30. Diken, Ö.E. and S. Özyalçın, OP-348 - Preoperative Incentive Spirometry Exercise Reduces the Risk of Atelectasis in Obese Cabg Patients. The American Journal of Cardiology, 2018. 121(8, Supplement): p. e26.

31. Moradian, S.T., A.A. Heydari, and H. Mahmoudi, What is the Role of Preoperative Breathing Exercises in Reducing Postoperative Atelectasis after CABG? Reviews on recent clinical trials, 2019. 14(4): p. 275-279.

32. Balandiuk, A.E. and I.A. Kozlov, Incentive spirometry for preoperative preparation of cardiac patients: 036. European Journal of Anaesthesiology (EJA), 2004. 21: p. 3-4.

33. Miskovic, A. and A.B. Lumb, Postoperative pulmonary complications. BJA: British Journal of Anaesthesia, 2017. 118(3): p. 317-334.

34. Carter, E.M. and H.W. Potts, Predicting length of stay from an electronic patient record system: a primary total knee replacement example. BMC medical informatics and decision making, 2014. 
14(1): p. 26.

35. Straub, D., M.-C. Boudreau, and D. Gefen, Validation guidelines for IS positivist research. Communications of the Association for Information systems, 2004. 13(1): p. 24.

36. Boudreau, M.-C., D. Gefen, and D.W. Straub, Validation in information systems research: A state-ofthe-art assessment. MIS quarterly, 2001: p. 1-16.

37. Lewis, B.R., C.A. Snyder, and R.K. Rainer Jr, An empirical assessment of the information resource management construct. Journal of Management Information Systems, 1995. 12(1): p. 199-223.

38. Association, W.M., World Medical Association Declaration of Helsinki: Ethical Principles for Medical Research Involving Human Subjects. JAMA, 2013. 310(20): p. 2191-2194.

39. Gilani, S.R.A., et al., Comparison of post-operative atelectasis in patients undergoing coronary artery bypass grafting with and without pre-operative incentive spirometry. Journal of Postgraduate Medical Institute (Peshawar-Pakistan), 2016. 30(2).

\section{Figures}




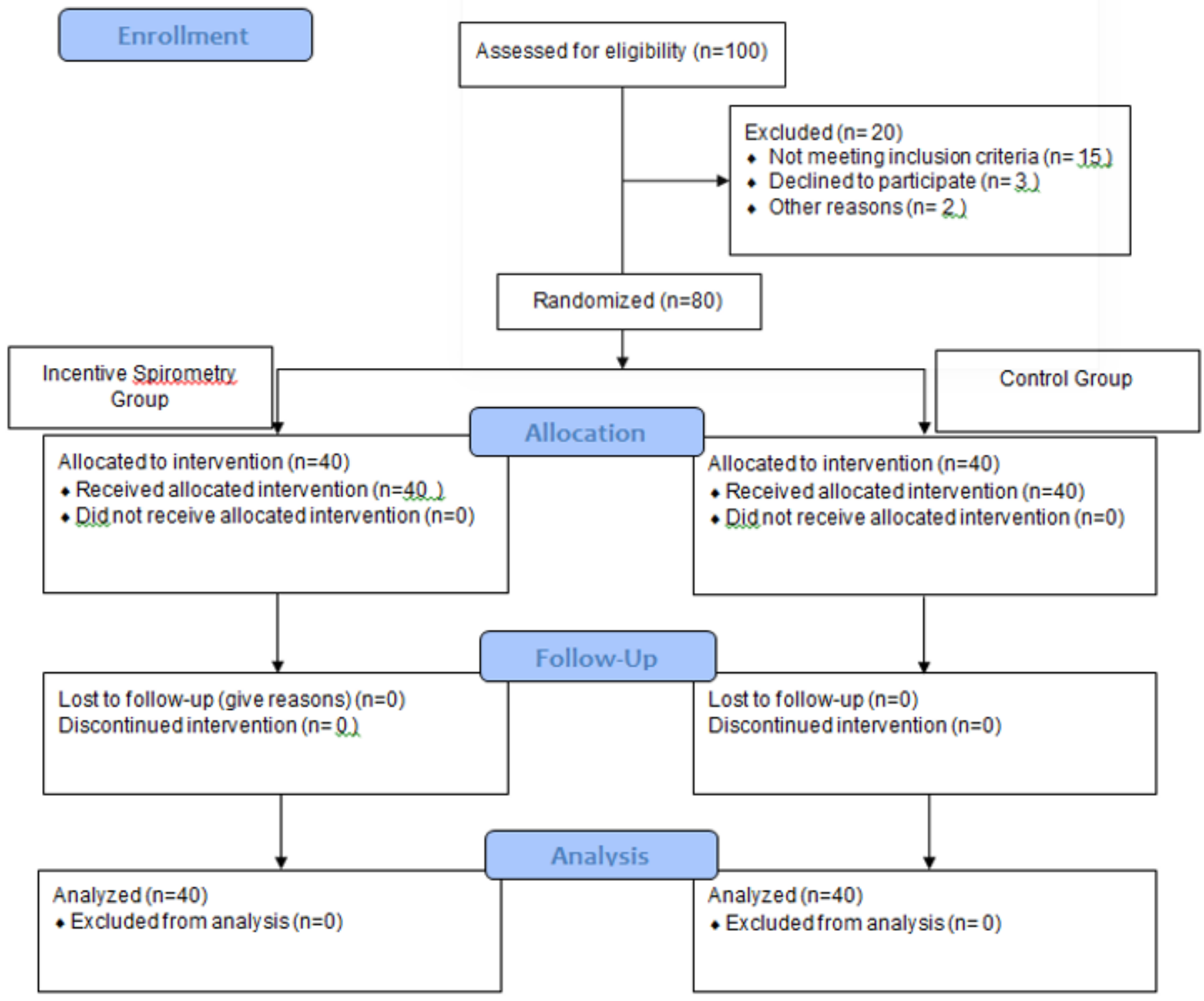

Figure 1

Consort diagram of patient screening and allocation 


\section{ATELECTASIS *}

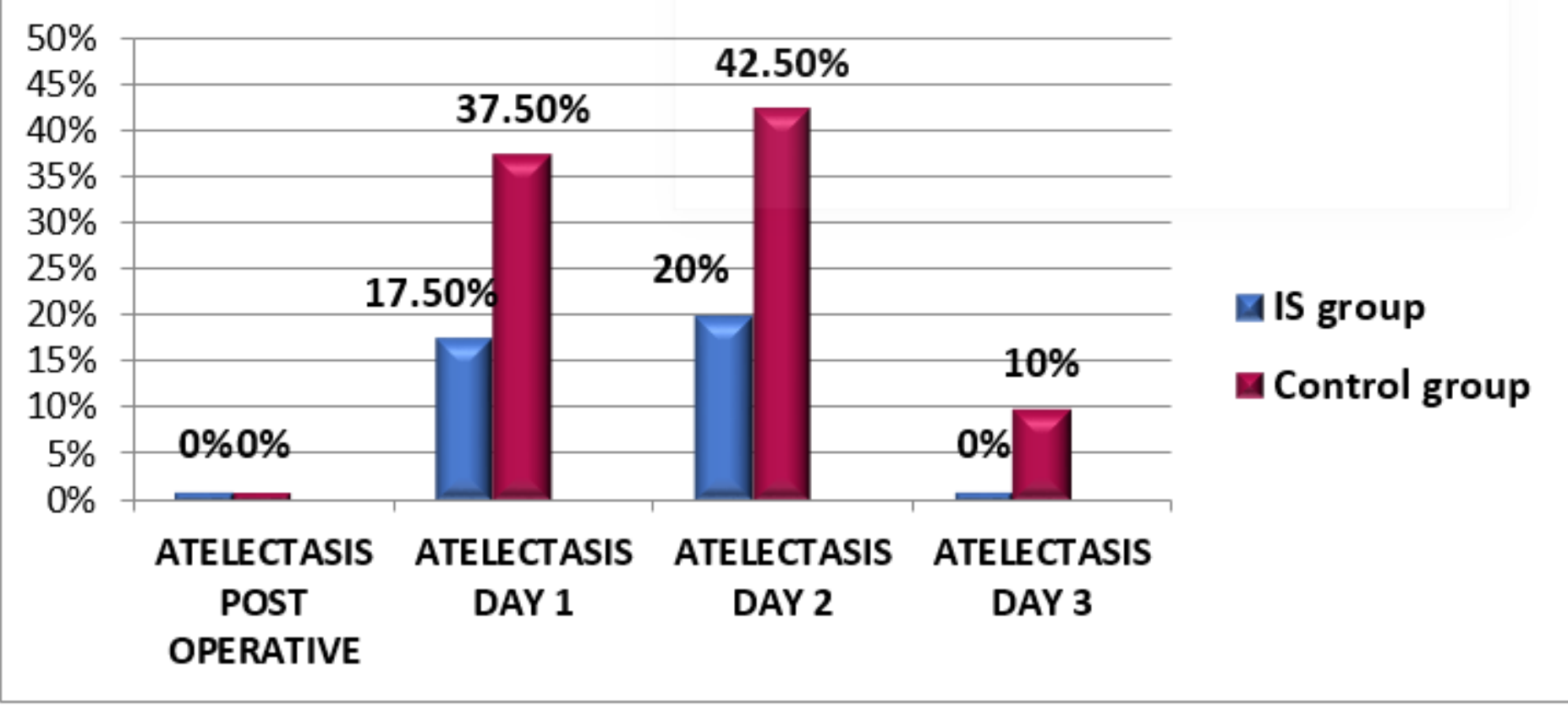

Figure 2

Percentage rate of atelectasis occurrence among IS and control groups. *: indicate p value of $\leq 0.05$

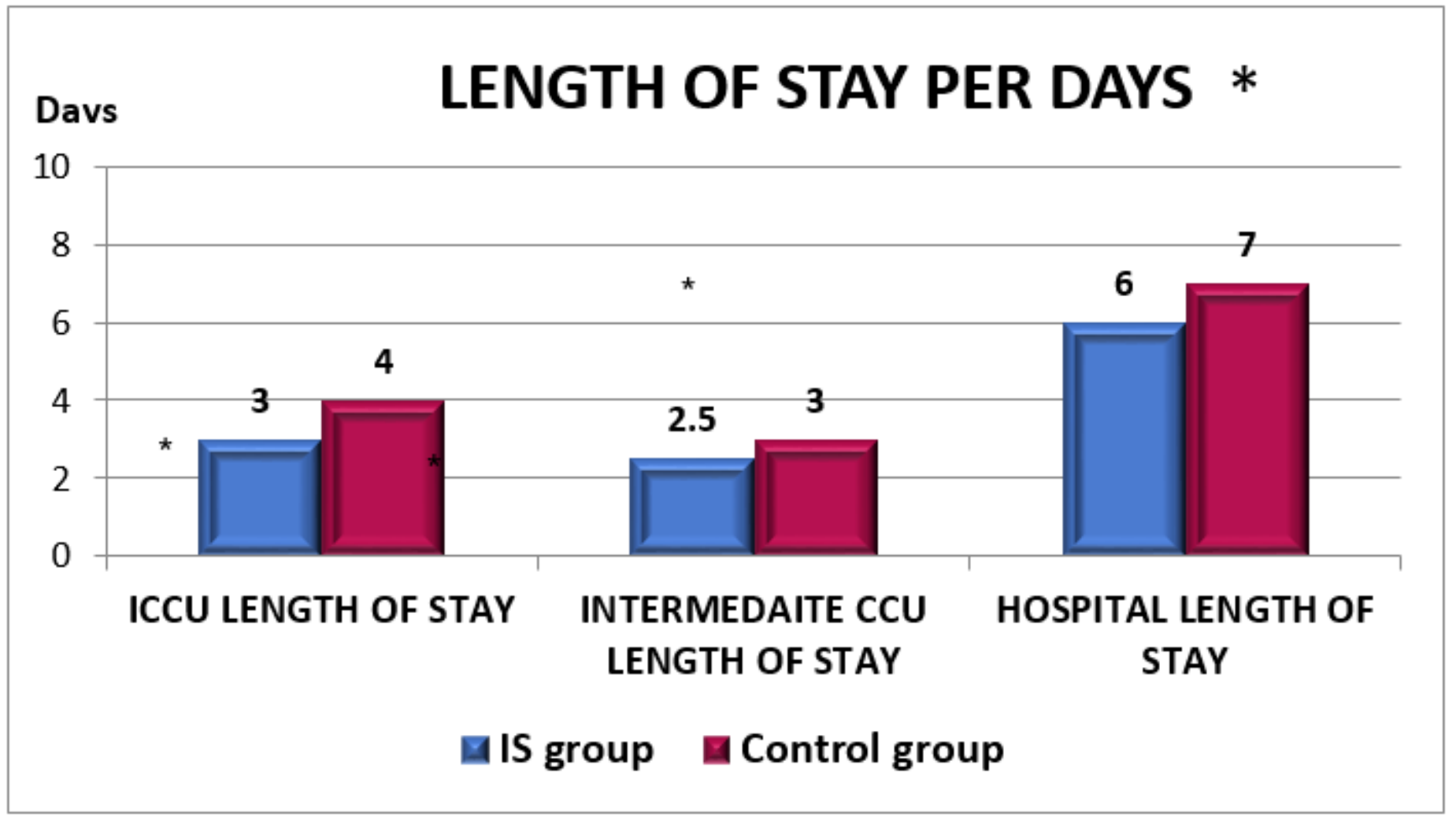

Figure 3 
Graphical comparison of median length of stay at hospital per day between IS and control group. *: indicate $p$ value of $\leq 0.05$

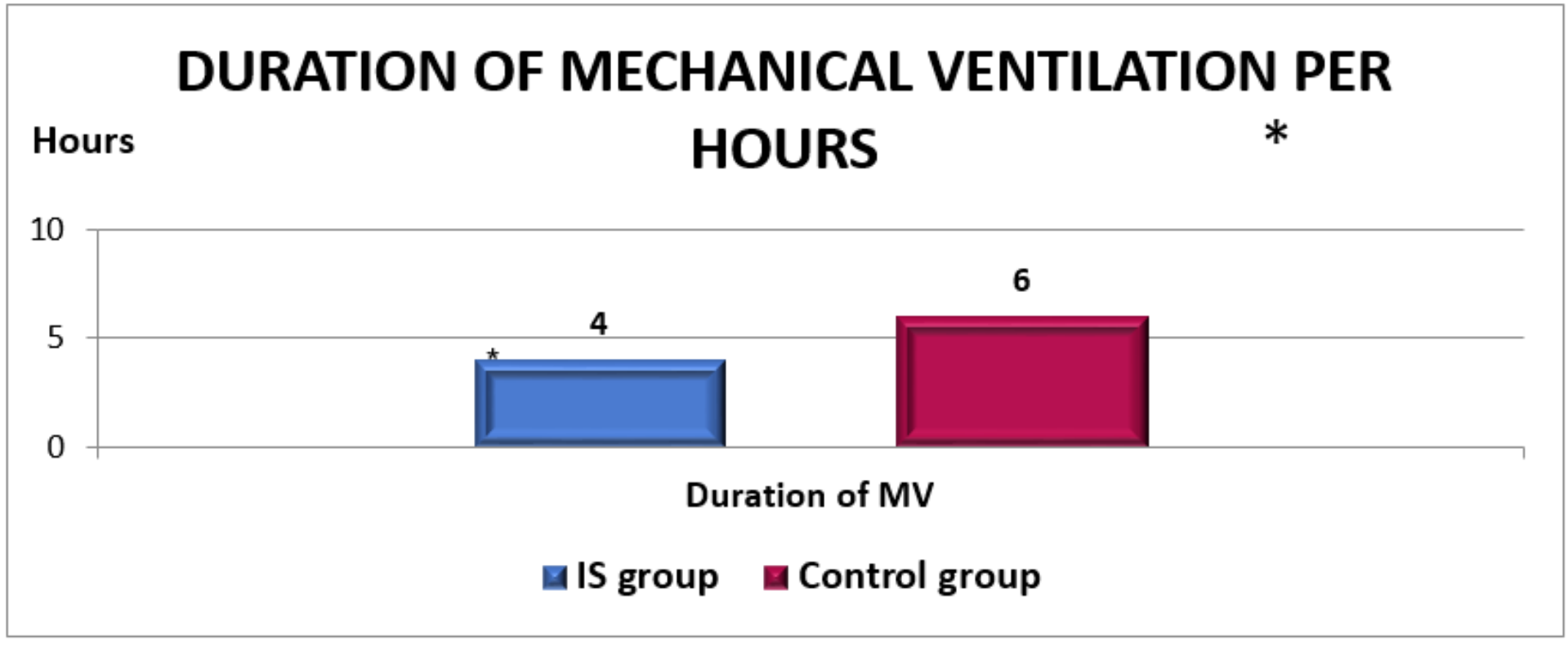

Figure 4

Graphical comparison of median duration of mechanical ventilation per hour between IS and control groups. *: indicate $p$ value of $\leq 0.05$

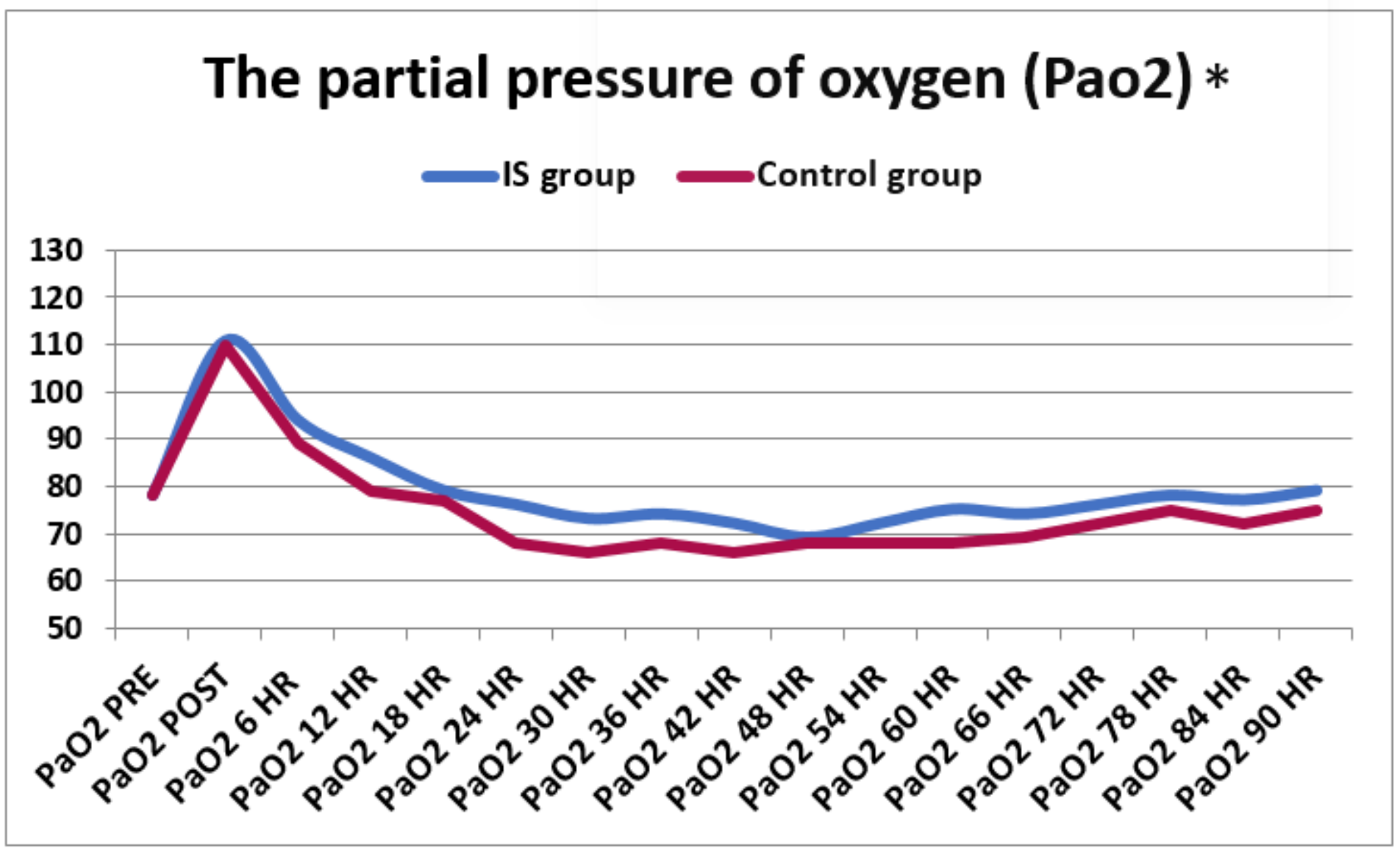

Figure 5 
Graphical comparison of median partial pressure of oxygen (Pao2) between IS and control groups. *: indicate $p$ value of $\leq 0.05$

\section{Oxygen saturation of arterial blood * (SaO2\%)}

IS group Control group

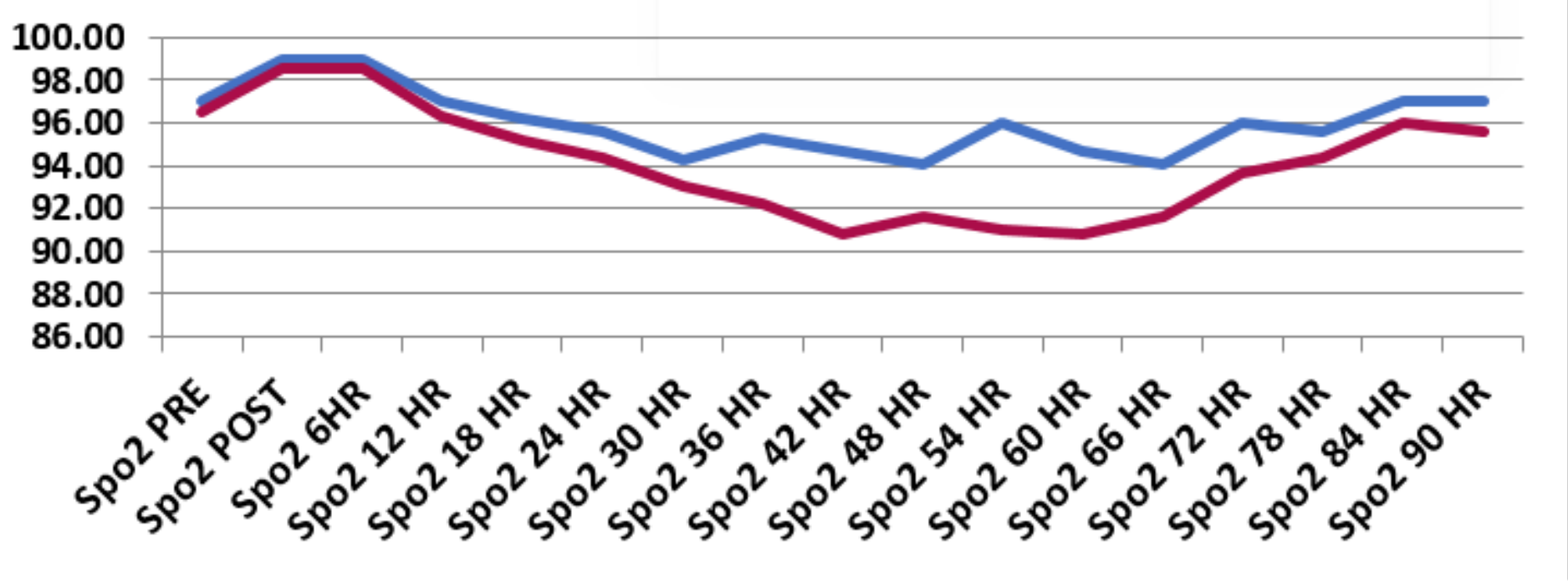

Figure 6

Graphical comparison of median oxygen saturation of arterial blood (SaO2\%) between IS and control groups. *: indicate $p$ value of $\leq 0.05$ 


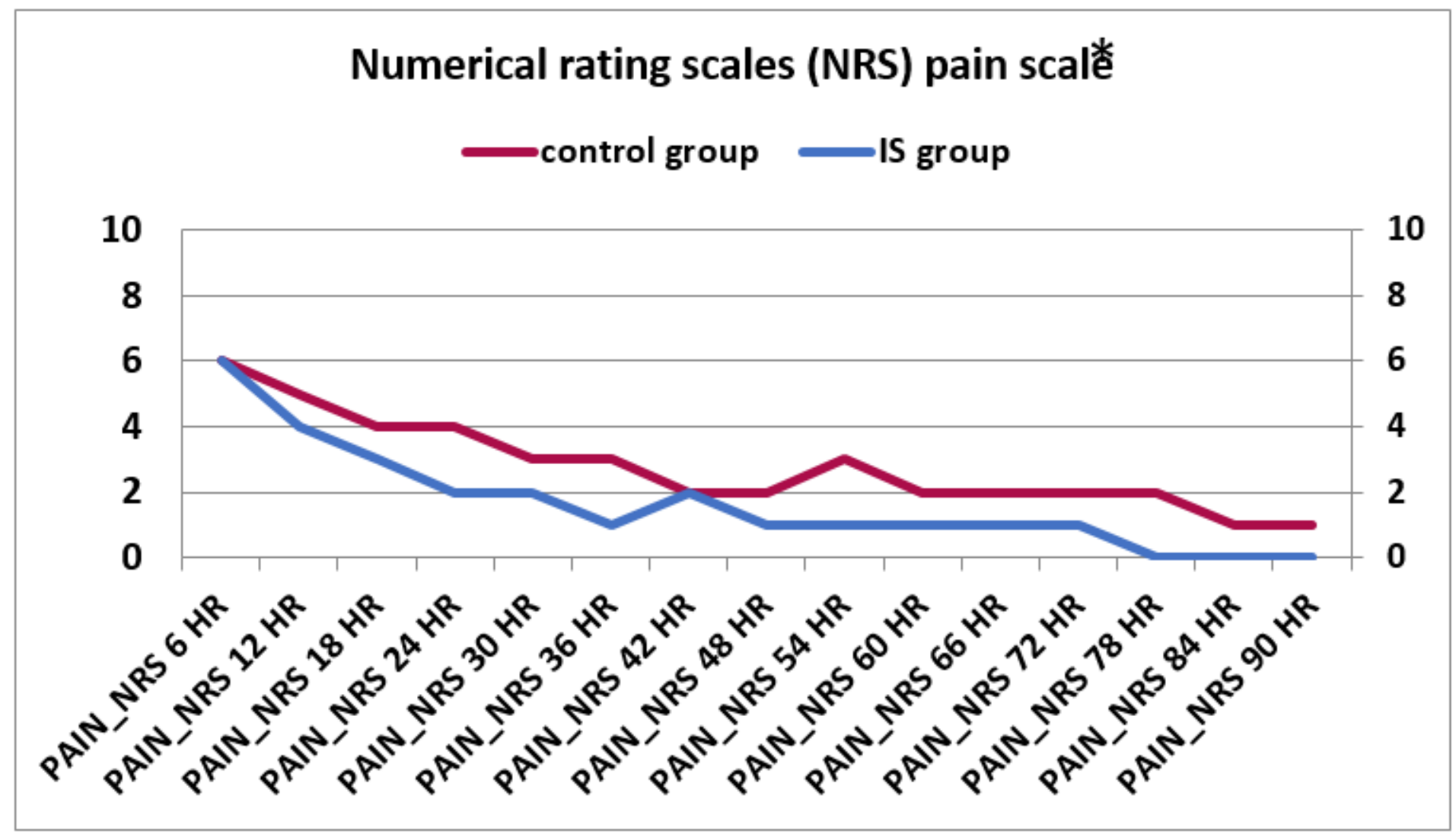

Figure 7

Graphical comparison of median numerical rating scale (NRS) pain scale between IS and control groups. *: indicate $p$ value of $\leq 0.05$ 